\title{
A phylogenetic study of endo-beta-1,4-glucanase in higher termites
}

\begin{abstract}
Cellulose is the most abundant polymer in the world and termites are the most important metazoan cellulose processors. Termites are divided into lower and higher termites, with the latter being the most derived and most specious. Although termites are known for their ability to digest wood, members of the family Termitidae (higher termites) are nutritionally diverse in their use of cellulose. This study investigated the evolution of endogenous cellulases in 25 species of higher termites, using phylogenetic inferences from mitochondrial (16S) and nuclear (28S) ribosomal RNA and endo- $\beta$-1,4-glucanase sequences. The translated endo- $\beta$ 1,4-glucanase amino acid order in all 41 sequences obtained showed high similarity to endo$\beta$-1,4-glucanases in the glycosyl hydrolase family 9 . The inferred endo- $\beta$-1,4-glucanase phylogenetic tree showed congruency with the mitochondrial/nuclear tree, with the fungusgrowers being the most basal group and the soil/litter- and wood/lichen/grass/litter-feeders being the most distal diphyletic feeding groups. The bacterial comb-grower formed a separate clade from the fungus-growers and is sister groups with the soil/litter- and wood/lichen/grass/litter-feeders. There was also a strong diphyletic relationship between endo- $\beta$-1,4-glucanases of upper layer soil-feeders and the other soil-feeders. Within the monophyletic wood/lichen/grass/litter-feeding termites' subclade, the nasutitermitines were polyphyletic and a strong diphyletic relationship was observed in the most distal lichen- and the grass/litter-feeders groups.
\end{abstract}

Keyword: $16 \mathrm{~S}$; 28S; Endo- $\beta$-1,4-glucanase; Termitidae 